\title{
Morphology-Dependent Ambient-Condition Growth of Perovskite Nanocrystals for Enhanced Stability in Photoconversion Device
}

Thanh-Hai Le ${ }^{1,2}$, Sanghyuck Lee ${ }^{2}$,Hyemi Jo' ${ }^{2}$, Ganghoon Jeong ${ }^{2}$, Mincheol Chang ${ }^{1,2} *$, and Hyeonseok Yoon $^{1,2} *$

${ }^{1}$ Alan G. MacDiarmid Energy Research Institute \& School of Polymer Science and Engineering, Chonnam National University, 77 Yongbong-ro, Gwangju 61186, South Korea

${ }^{2}$ Department of Polymer Engineering, Graduate School, Chonnam National University, 77 Yongbong-ro, Gwangju 61186, South Korea

Corresponding Authors:

*E-mail: hyoon@chonnam.ac.kr; mchang35@chonnam.ac.kr 


\section{EXPERIMENTAL SECTION}

Materials. Oleic acid (90\%) was purchased from Tokyo Chemical Industry. Octadecene (90\%), cesium carbonate $\left(\mathrm{Cs}_{2} \mathrm{CO}_{3}, 99.9 \%\right)$, oleylamine $(70 \%)$, hexane $(99.9 \%)$, lead(II) bromide $\left(\mathrm{PbBr}_{2}, 99.99 \%\right)$, and ethyl acetate (99.8\%) were purchased from Aldrich. Cesium oleate $(74 \mathrm{mM})$ was obtained by dissolving $0.4 \mathrm{~g}$ of $\mathrm{Cs}_{2} \mathrm{CO}_{3}$ in $15 \mathrm{~mL}$ of octadecene and 1.5 $\mathrm{mL}$ of oleic acid at $150{ }^{\circ} \mathrm{C}$ under inert conditions.

Synthesis of CPNCs with different morphologies. 2D and 3D CPNCs were synthesized using a hot injection method. ${ }^{1}$ Briefly, $69 \mathrm{mg}$ of $\mathrm{PbBr}, 0.5 \mathrm{~mL}$ of oleylamine, $0.5 \mathrm{~mL}$ of oleic acid, and $5 \mathrm{~mL}$ of octadecene were mixed in a three-neck reactor and heated to $100{ }^{\circ} \mathrm{C}$ under a vacuum environment for $30 \mathrm{~min}$. Then, the reactor was further heated to $120{ }^{\circ} \mathrm{C}$ until a clear solution was obtained. At $120^{\circ} \mathrm{C}, 0.3 \mathrm{~mL}$ of a hot $74 \mathrm{mM}$ cesium oleate solution was swiftly injected and maintained for $5 \mathrm{~s}$ to grow nanoplatelets. The 3D nanocubes were synthesized in a similar manner with a reaction temperature of $185^{\circ} \mathrm{C}$ instead of $120^{\circ} \mathrm{C}$. All high-quality CPNCs were purified once using hexane/ethyl acetate and finally dispersed in hexane for further use.

Fabrication of photoconversion devices. A microelectrode with dimensions of $50 \mu \mathrm{m} \times 2$ $\mathrm{mm}$ (channel width $\times$ length) was fabricated via the deposition of a $50 \mathrm{~nm} \mathrm{Au} / \mathrm{Cr}$ layer on a $\mathrm{SiO}_{2} / \mathrm{Si}$ substrate using thermal evaporation through a shadow mask. The photoconversion devices were fabricated by spin-coating a $20 \mu \mathrm{L} \mathrm{CPNC}$ solution onto the microelectrode at $2000 \mathrm{rpm}$ for $60 \mathrm{~s}$. All deposition processes were performed in air with a relative humidity of $40-50 \%$ at $25{ }^{\circ} \mathrm{C}$. Finally, the devices were stored in a dark cabinet with a relative humidity of $30 \%$ at $25^{\circ} \mathrm{C}$. 
Photocurrent monitoring. A homemade measurement setup was built for photocurrent monitoring under UV irradiation. The photoresponsivity of the photoconversion devices was measured using a semiconductor parameter analyzer (Keithley 2636A). Light irradiation was achieved with a $365 \mathrm{~nm}$ UV light source at a power density of $4.2 \mathrm{~mW} \mathrm{~cm}^{-2}$. The output characteristics of the devices were measured under an applied voltage of $3.0 \mathrm{~V}$ at $25^{\circ} \mathrm{C}$ in air.

Characterization. X-ray diffraction (XRD) of the PNC samples was performed using a Rigaku X-ray diffractometer (D/MAX-2500, Japan) with $\mathrm{Cu} \mathrm{K \alpha}$ radiation $(\lambda=1.5418 \AA)$ at $45 \mathrm{kV}$ and $55 \mathrm{~mA}$. The transmission electron microscopy (TEM) images, high-angle annular dark-field scanning TEM (HAADF-STEM) image, and element mappings were acquired using a JEOL JEM-2100F instrument. The optical absorption spectra were collected using an Optizen 2120UV spectrophotometer (Mecasys, Korea). Photoluminescence (PL) spectra were measured using a Hitachi F-4500 fluorescence spectrophotometer (Japan) under excitation at $450 \mathrm{~nm}$ with a $20 \mathrm{~ms}$ integration time and $2.5 \mathrm{~nm}$ slit width. Time-resolved PL (TRPL) measurements were performed using a time-correlated single-photon counting spectrofluorometer with a pulsed laser diode $(\lambda=450 \mathrm{~nm})$. Absolute PL quantum yields (QYs) were recorded on an integrating sphere setup (C9920-02, Hamamatsu Photonics, Japan). High-performance X-ray photoelectron spectroscopy (XPS) was conducted using a K$\mathrm{Alpha}^{+}$spectrometer equipped with an $\mathrm{Al} \mathrm{K} \alpha \mathrm{X}$-ray source $(12 \mathrm{keV})$ (Thermo Fisher Scientific, USA).

Numerical calculations. Three different perovskite nanoplatelet sizes were modeled: a small nanoplatelet $(\mathrm{P} 1,1 \times 1 \times 1)$, two merged nanoplatelets $(\mathrm{P} 2,1 \times 2 \times 1)$, and three merged nanoplatelets $(\mathrm{P} 3,2 \times 2 \times 1)$ formed by extending the orthorhombic-phase $\mathrm{CsPbBr} 3$ unit cell along the $x$ - and $y$-directions. All DFT calculations were carried out using the pseudopotential plane-wave method in the Quantum Expresso software package. ${ }^{2-3}$ The exchange-correlation 
energy was described by the generalized gradient approximation (GGA) using the PerdewBurke-Ernzerhof (PBE) functional. ${ }^{4}$ The ultrasoft pseudopotential with a cutoff energy of 46 Ry and cutoff charge density of 420 Ry was used for band structure and density of states (DOS) calculations. A $4 \times 4 \times 4$ Monkhorst-Pack mesh was employed for self-consistent field and DOS calculations 

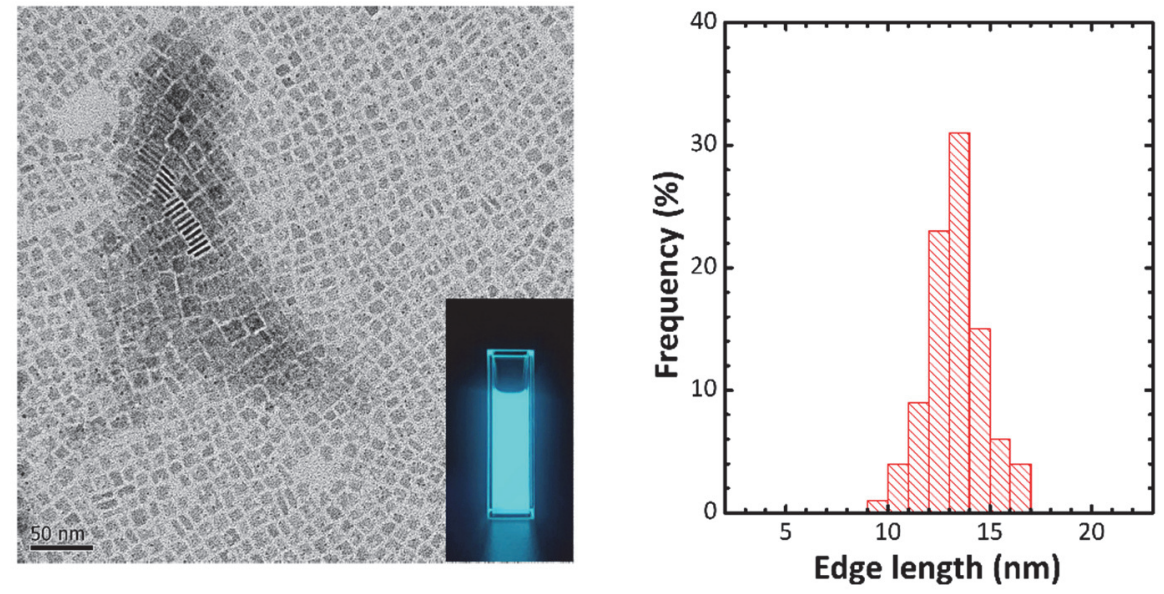

Figure S1. Histogram showing the particle size distribution of the nanoplatelets. 

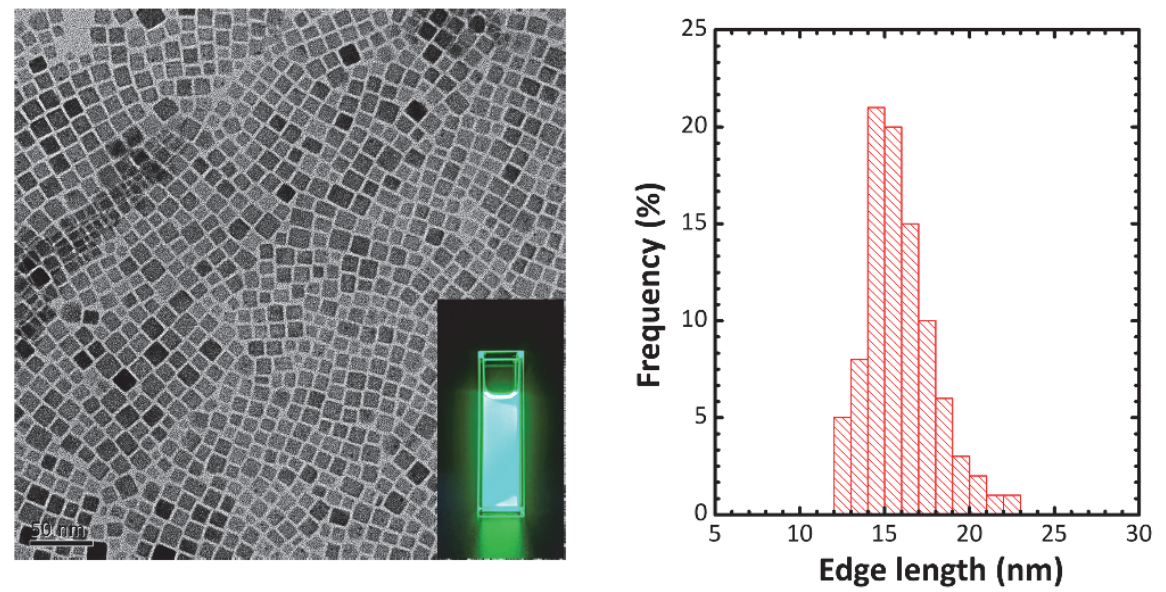

Figure S2. Histogram showing the particle size distribution of the nanocubes. 

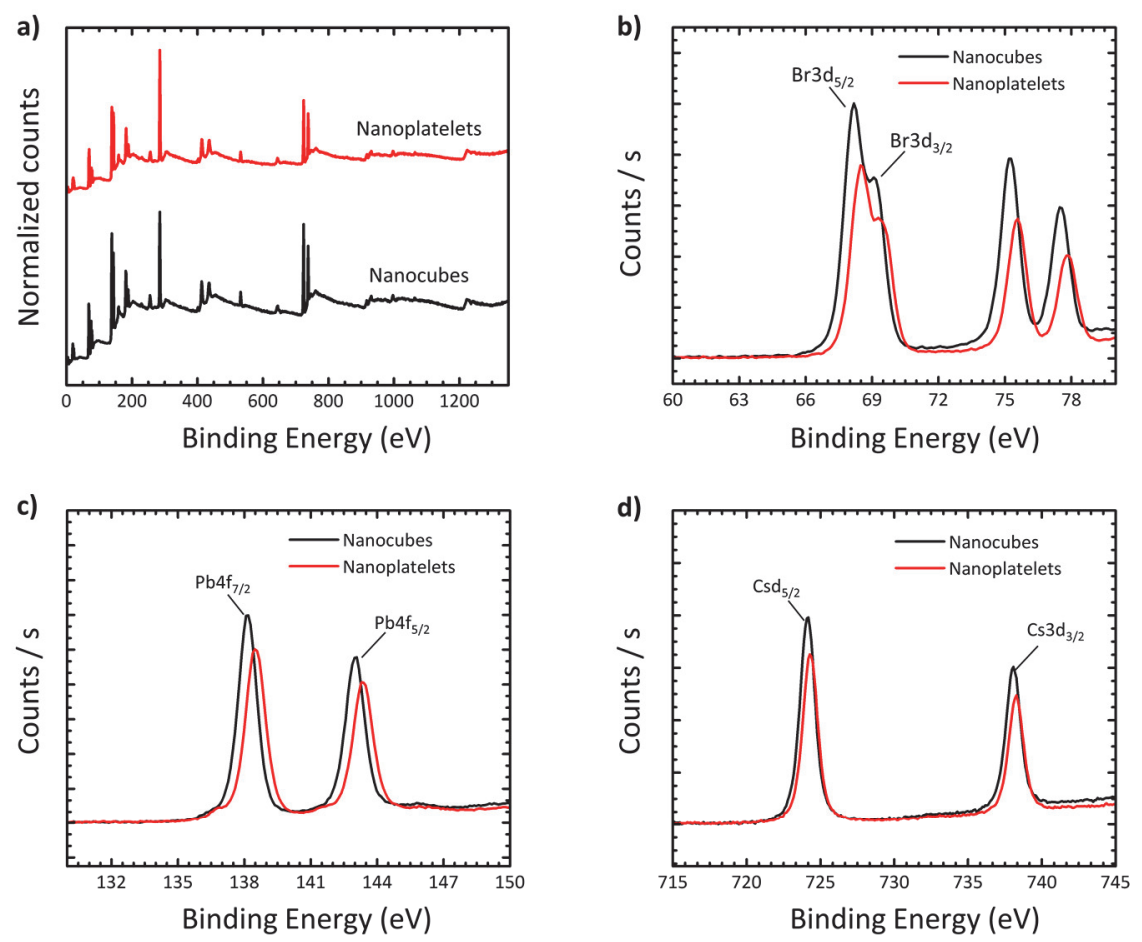

e)

\begin{tabular}{ccc}
\hline Atomic (\%) & Nanoplatelets & Nanocubes \\
\hline $\mathrm{Br}$ & 69.57 & 72.25 \\
$\mathrm{~Pb}$ & 14.18 & 11.05 \\
$\mathrm{Cs}$ & 16.25 & 16.70 \\
\hline
\end{tabular}

Figure S3. (a) XP spectra of the nanoplatelets and nanocubes. High-resolution XPS analyses corresponding to (b) $\mathrm{Br} 3 \mathrm{~d}$, (c) $\mathrm{Pb} 4 \mathrm{f}$, and (d) Cs 3d of the nanoplatelets and nanocubes. (e) Atomic ratios determined via XPS of the nanoplatelets and nanocubes. 


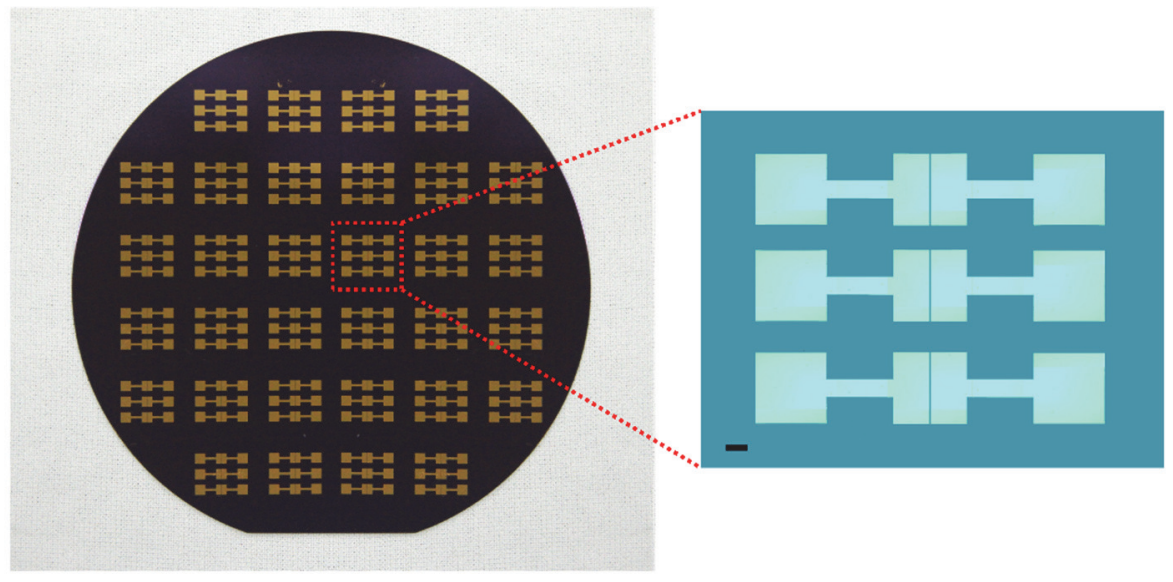

Figure S4. Optical image of a microelectrode (scale bar is $100 \mu \mathrm{m}$ ). 
a)

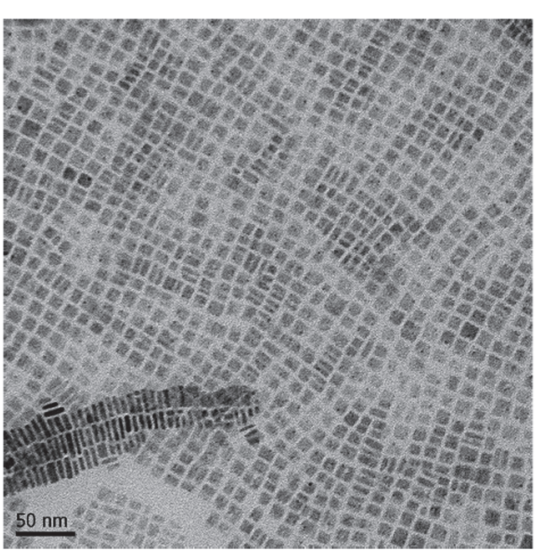

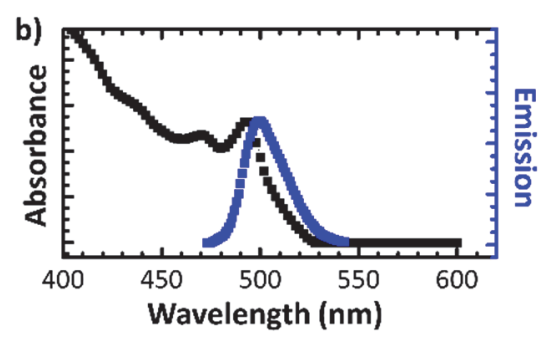

c)

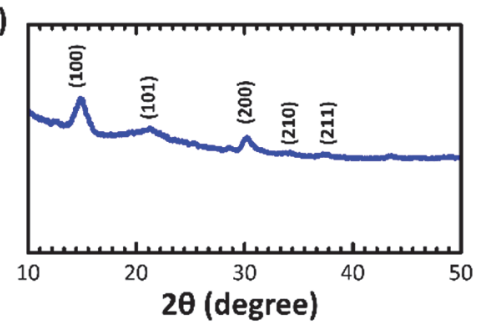

Figure S5. TEM image and optical properties of the thicker nanoplatelets (control) synthesized at $135^{\circ} \mathrm{C}$. 
a)
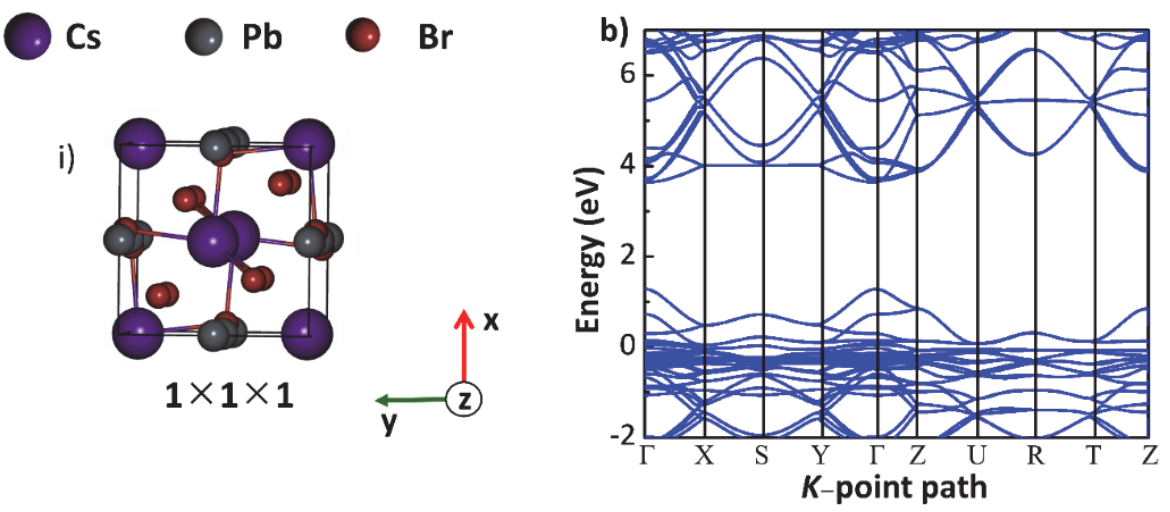

ii)
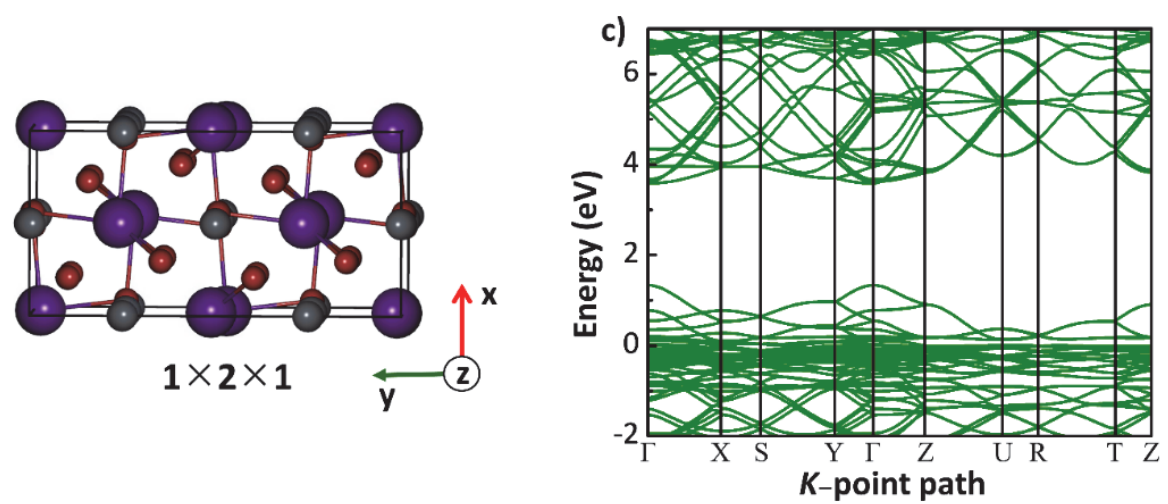

iii)
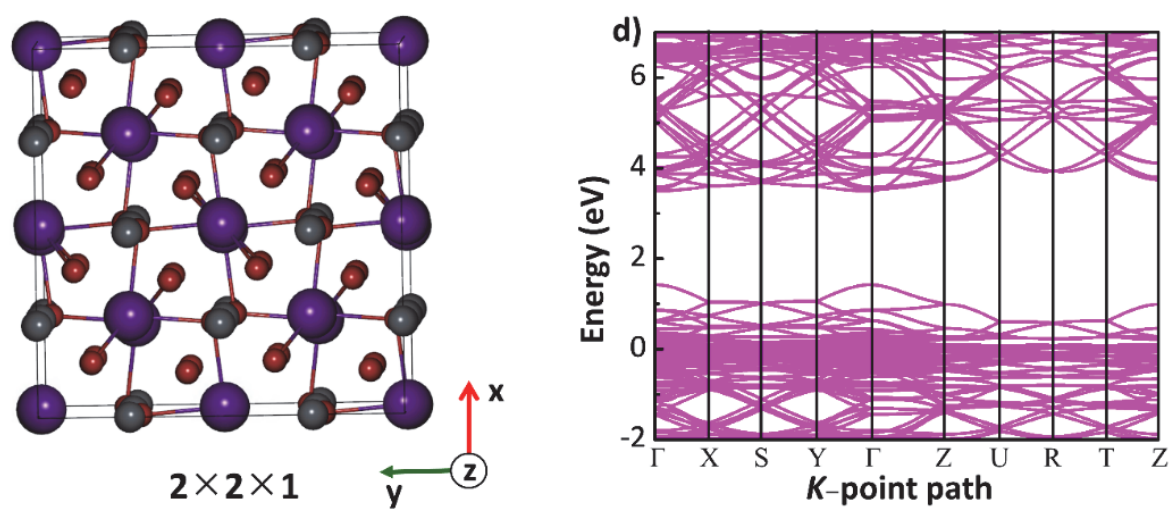

Figure S6. Calculated electronic properties of cubic-phase $\mathrm{CsPbBr} 3$ with different sizes along the $y$-direction. (a) Miniature nanoplatelet models: (i) small nanoplatelet $(\mathrm{P} 1,1 \times 1 \times 1)$, (ii) two merged nanoplatelets $(\mathrm{P} 2,1 \times 2 \times 1)$, and (iii) three merged nanoplatelets $(\mathrm{P} 3,2 \times 2 \times 1)$. (b, c, d) Corresponding electronic band structures determined using the GGA/PBE functional. 


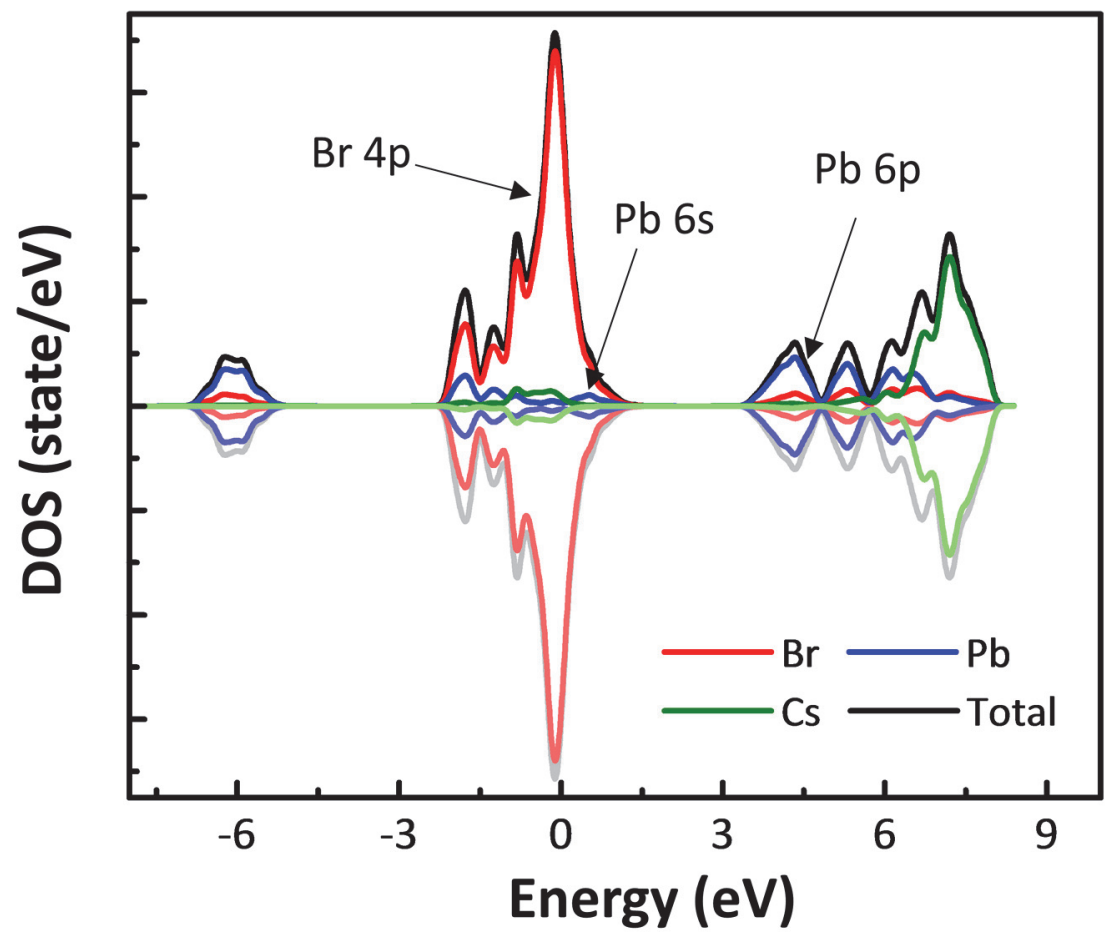

Figure S7. Detail DOSs of orthorhombic-phase $\mathrm{CsPbBr}_{3}$ (P1 model). 

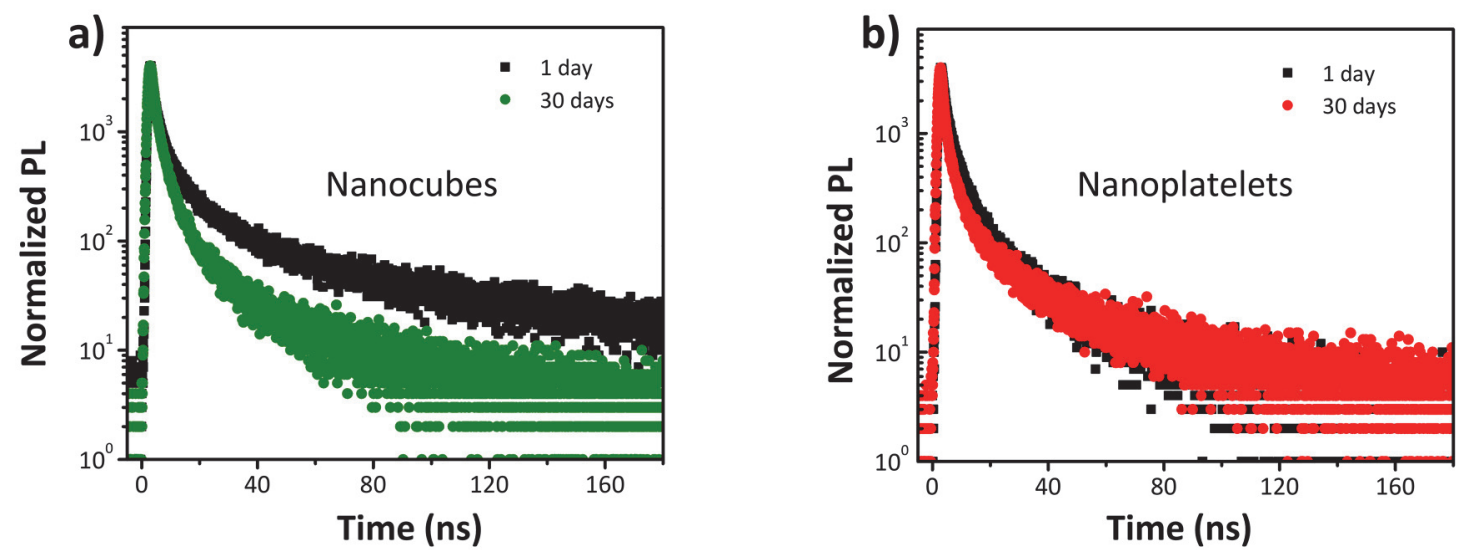

Figure S8. Time-resolved PL decay curves of a) nanocubes and b) nanoplatelets after aging. 

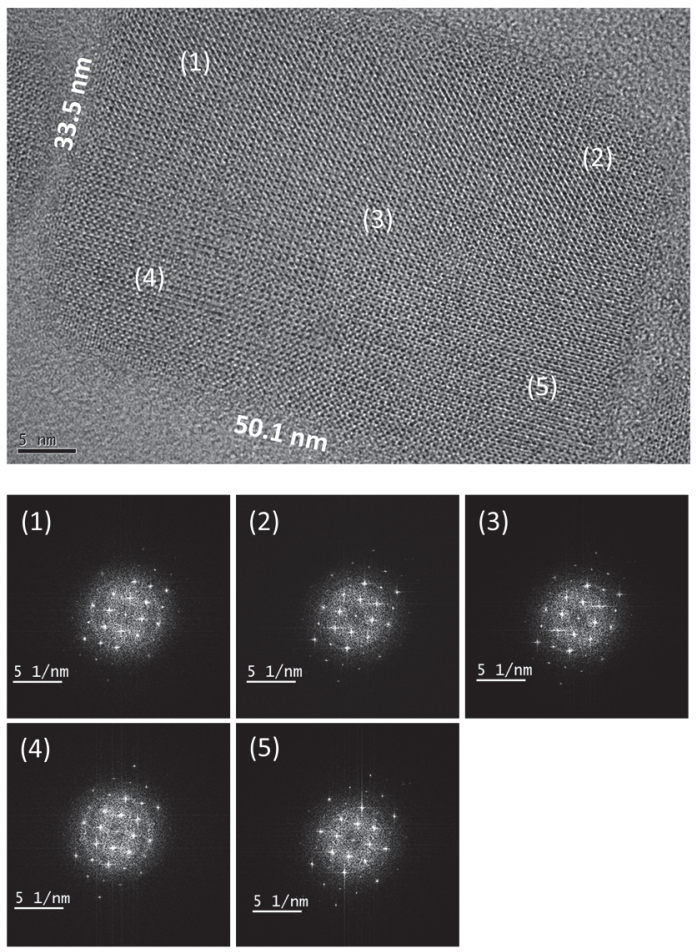

Figure S9. High-resolution TEM and FFT images of a nanoplatelet $(33.5 \times 50.1 \mathrm{~nm})$ formed from facet-to-facet attachment of neighboring nanoplatelets. 


\section{REFERENCES}

(1) Protesescu, L.; Yakunin, S.; Bodnarchuk, M. I.; Krieg, F.; Caputo, R.; Hendon, C. H.; Yang, R. X.; Walsh, A.; Kovalenko, M. V. Nanocrystals of cesium lead halide perovskites $\left(\mathrm{CsPbX}_{3}, \mathrm{X}=\mathrm{Cl}, \mathrm{Br}\right.$, and I): Novel optoelectronic materials showing bright emission with wide color gamut. Nano Lett. 2015, 15 (6), 3692-3696.

(2) Giannozzi, P.; Baroni, S.; Bonini, N.; Calandra, M.; Car, R.; Cavazzoni, C.; Ceresoli, D.; Chiarotti, G. L.; Cococcioni, M.; Dabo, I.; Dal Corso, A.; de Gironcoli, S.; Fabris, S.; Fratesi, G.; Gebauer, R.; Gerstmann, U.; Gougoussis, C.; Kokalj, A.; Lazzeri, M.; Martin-Samos, L.; Marzari, N.; Mauri, F.; Mazzarello, R.; Paolini, S.; Pasquarello, A.; Paulatto, L.; Sbraccia, C.; Scandolo, S.; Sclauzero, G.; Seitsonen, A. P.; Smogunov, A.; Umari, P.; Wentzcovitch, R. M. QUANTUM ESPRESSO: A modular and open-source software project for quantum simulations of materials. J. Phys. Condens. Matter 2009, 21 (39), 395502.

(3) Kresse, G.; Furthmüller, J. Efficient iterative schemes for ab initio total-energy calculations using a plane-wave basis set. Phys. Rev. B 1996, 54 (16), 11169-11186.

(4) Perdew, J. P.; Burke, K.; Ernzerhof, M. Generalized gradient approximation made simple. Phys. Rev. Lett. 1996, 77 (18), 3865-3868. 